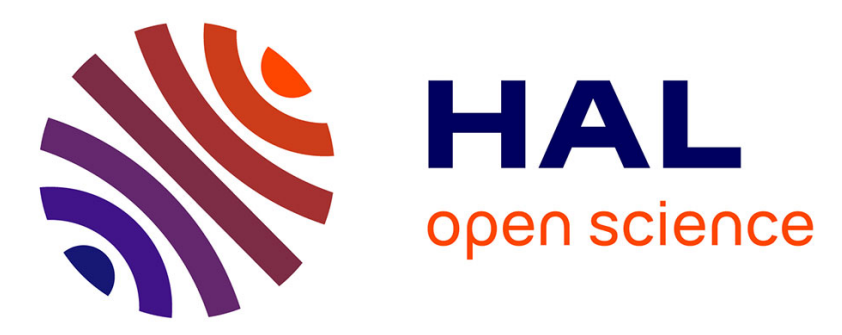

\title{
L'immersion par corps des chercheurs. Expérimentations méthodologiques à Salvador da Bahia et Caracas
}

\author{
Sandra Fiori, Rachel Thomas
}

\section{To cite this version:}

Sandra Fiori, Rachel Thomas. L'immersion par corps des chercheurs. Expérimentations méthodologiques à Salvador da Bahia et Caracas. Corps: Revue interdisciplinaire, 2016, Quels corps demain?, 14, pp.229-237. 10.3917/corp1.014.0229 . hal-01346847

\section{HAL Id: hal-01346847 \\ https://hal.univ-grenoble-alpes.fr/hal-01346847}

Submitted on 19 Jul 2016

HAL is a multi-disciplinary open access archive for the deposit and dissemination of scientific research documents, whether they are published or not. The documents may come from teaching and research institutions in France or abroad, or from public or private research centers.
L'archive ouverte pluridisciplinaire HAL, est destinée au dépôt et à la diffusion de documents scientifiques de niveau recherche, publiés ou non, émanant des établissements d'enseignement et de recherche français ou étrangers, des laboratoires publics ou privés.

\section{(1) (1) $\$$}

Distributed under a Creative Commons Attribution - NonCommercial - NoDerivatives $\mid 4.0$ 


\section{L'IMMERSION PAR CORPS DES CHERCHEURS EXPERIMENTATIONS METHODOLOGIQUES À SALVADOR DA BAHIA ET CARACAS}

Sandra FIORI \&Rachel THOMAS

Pour citer cet article :

Fiori Sandra \&Thomas, Rachel (2016). «L'immersion par corps des chercheurs. Expérimentations méthodologiques à Salvador da Bahia et Caracas », Corps : revue interdisciplinaire, $\mathrm{n}^{\circ} 14$, Paris, CNRS Éditions, pp. 229-237.

\section{INTRODUCTION}

En quoi l'immersion répétée, mais de courte durée, dans des terrains problématiques participe-t-elle d'une transformation des postures du chercheur et de ses modes de production des données ? Qu'est-ce que la prise en compte des états de corps du chercheur fait à l'approche sensible de l'urbanité contemporaine ?

Ces questions ont traversé une recherche $\mathrm{ANR}^{1}$ qui a fait collaborer durant quatre ans des chercheurs français, brésiliens et vénézuéliens, issus de l'urbanisme, de la sociologie et de la danse. Son objectif était de déchiffrer les énigmes sensibles liées à l'expérience piétonne "sous contrainte », dans un contexte d'évolution des politiques d'aménagement liées à la mobilité urbaine : complexification des espaces et de leur gestion, prégnance du paradigme de la fluidité, ambiguïtés de l'idéologie de la pacification. Nous faisions l'hypothèse qu'en changeant, ce contexte affecte les pratiques citadines et engage des formes particulières de se mouvoir et d'être-ensemble en public. Enquêtant sur des espaces problématiques à différents niveaux (spatial, social, sensible), nous avons cherché à décrire comment s'orchestre leur partage, les types de savoir-être mobilisés par le piéton, la manière dont ces savoirs composent avec les ressources matérielles et sensibles des lieux. Les démarches de terrain mises en œuvre empruntent à l'ethnographie, mais procèdent d'expérimentations et d'adaptation aux contextes investigués. Ce sont ces expérimentations méthodologiques, et la façon dont en éprouvant les corps des chercheurs elles ont transformé leurs analyses, que nous explicitons ici.

\section{LE PARTAGE DE L'ESPACE PUBLIC SUR DES TERRAINS SUD-AMERICAINS}

Deux terrains ont été investigués : la Redoma de Petare à Caracas (Venezuela) et Porto da Barra à Salvador de Bahia (Brésil). Spatialement complexes, fortement motorisés, soumis à des projets de réaménagements polémiques, ces lieux questionnent la place de chacun dans l'espace. Empreints d'une violence ordinaire et d'une insécurité latente, ils accueillent un mouvement dense et permanent au cœur de nombreux conflits d'usage. Hyperesthésiques, ils saturent le piéton de sollicitations sensibles éprouvant les corps et les attentions.

La Redoma de Petare,véritable noeud de transports, dessert l'Est de la capitale vénézuélienne et les barrios de Petare, ensemble de quartiers auto-construits de près d'un million d'habitants. A leur pied, au carrefour de grandes avenues, le secteur comprend une station de métro, des terminaux de bus publics, une centaine de lignes de 
minibus du secteur artisanal et une ligne de train aérienne. La densité du trafic, le manque de planification et les dysfonctionnements des transports soumettent le citadin aux épreuves d'un environnement labile, où l'incertain, l'imprévu, l'incident constituent des dimensions structurantes de l'expérience ordinaire.La Redoma est aussi une vaste zone marchande, avec ses boutiques implantées le long des avenues et ses dédales de marchés couverts vendant toutes sortes de produits à des prix attractifs. Cette présence de commerces formels et les dizaines de milliers de citadins qui transitent chaque jour ont attiré plus de deux millevendeurs de rue (buhoneros),faisant de la Redoma un immense marché, dense et surchargé de sollicitations. Installés de manière quasipermanente, les buhorenoscolonisent le moindre interstice et débordent sur la chaussée, jusqu'à entraver un trafic motorisé déjà saturé et à perturber le cheminement sur les trottoirs.Le partage de l'espace,posé sous les termes de la «récupération » d'un espace public «envahi » par les vendeurs de rue, constitue alors un enjeu majeur pour les institutions locales qui, en réponse, ont créé un nouveau marché couvert (devant accueillir et régulariser plus de sept cent vendeurs ambulants)et prévu de réaménager un kilomètre et demi de trottoirs (censés être ainsi libérés des vendeurs délocalisés vers le marché couvert). Dans les faits, début 2014,la récupération des trottoirs a démarré avant l'ouverture du marché : profitant de la trêve des commerçants après les fêtes de fin d'année, les bulldozers ont en quelques jours retourné les trottoirs de la Redoma. Or au printemps, la plupart des vendeurs s'étaient réinstallés parmi les gravats, évoquant un quasi paysage de guerre et une territorialisation à la mesure des tensions entre les buhoneros et la municipalité. La question des épreuves auxquelles est soumis le piéton s'avère donc ici liée à celle d'une conflictualité socio-politique sous-jacente.

Cette conflictualité colore également l'expérience piétonne ordinaire à Porto da Barra ${ }^{2}$. Les clivages sociaux se lisent dans la rue dans les contrastes d'apparences corporelles (peaux claires/mates, apprêt vestimentaire) et de modes de déplacement (voiture climatisée/à pied). Mais ici, la dimension politique devient plus explicite depuis l'intensification des processus de pacification.

Le quartier, ouvert sur la Baie de tous les Saints, accueille de nombreuses pousadas (pension de famille) et des immeubles d'habitation affichant leur standing par la sophistication de leur système de protection (murs, grilles, barbelés, vigiles). Prèsde ces espaces résidentiels, depuis desfavelas affluent quotidiennement dans la rue des milliers de travailleurs pauvres. Or Barra, traversé par deux avenues routières, subitun trafic motorisé dense. Bus de tourisme et privés, voitures, taxis se disputent la voirie, sans égard pour les piétons. Nombreux, ceux-ci slaloment pour rejoindre les commerces, restaurants et arrêts de bus alignés face à la mer. Outre la densité de véhicules et de population, ils ont à gérer des défauts d'aménagements, la présence des commerçants ambulants, le poids d'objets poussés, tirés ou portés. Ceux qui rejoignent en contrebas la plage urbaine le font avec plus d'indolence et de lenteur. Quant aux enfants des rues, ils déambulent sur des territoires délimités par leurs trafics. La nuit, la présence des habitants des rues, des trafiquants et consommateurs de crack oblige chacun à des détours et rend certains espaces inaccessibles.Le partage de l'espace a donc ici à voir avec une violence latente qui s'exerce entre les différentes couches de la population (contrastant avec l'apparente chaleur des échanges) et dans la dispute des territoires. Pour endiguer cette violence, à l'aube d'évènements sportifs mondiaux (Copa 2014, JO 2016), le pays adopte des politiques de pacification ${ }^{3}$ basées surles principes suivants : reconquête des territoires, esthétisation des quartiers, restauration de l'ordre public,construction d'une conscience publique. A Barra, depuis 2013, des requalifications majeures transforment le paysage : réaménagement des espaces de 
circulation, disparition du pavage portugais (lourd de sens mémoriel), renforcement de la signalétique au sol et de l'éclairage public, sensibilisation à la propreté de la rue. Depuis 2010, nous assistons aussi à un déploiement conséquent de la police militaire, à un contrôle du commerce ambulant et à un rejet en périphérie des populations démunies, montrant que les politiques de pacification désignent aussi une tendance à anéantir - au nom d'un mieux vivre-ensemble - toute velléité de conflit dans les espaces publics urbains, conflit pourtant au cœur de la vie publique.Entre la nécessité de temporiser une violence urbaine toujours latente sinon grandissante, et celle d'instituer un nouvel ordre public en ville, "pacifier »révèle ainsi une ambivalence : celle qui consiste à mieux maîtriser l'espace du mouvement, à encadrer les corps ainsi qu'à instituer des formes d'écart, de retrait, de repli. A Salvador mais aussi à Caracas, cette ambivalence nous poseune double question, conceptuelle et méthodologique. Dans cette dichotomie à l'œuvre, comment se déploie l'être-ensemble ? Parce que l'être-ensemble se joue souvent en deçàdu langage, dans des attitudes et des affects, comment l'observer et le décrire?

\section{IN-SITU : LE CORPS DU CHERCHEUR DESACCESSOIRISE}

De telles problématiques interrogent la façon dont des processus s'incarnent dans le quotidien du piéton. Sur chaque terrain, elles nous ont conduit à engager des collectifs de chercheurs dans diverses marches urbaines. Si cette démarche relève d'une ethnographie sensible pratiquée dans notre laboratoire, où la marche constitue un instrument de compréhension des relations sensibles à l'environnement, la spécificité méthodologique est ici triple. Le décadrage sur des terrains au Sud, peu investis par l'équipe, marqués socialement et impactés par des questions sécuritaires, a immédiatement nécessité que nous adaptions nos postures d'immersion : d'appareillés, nous avons dû nous « désaccessoiriser » et faire de nos corps les principaux instruments de collecte des données in situ (Thomas \& al., 2010). Le statut de la marche, embrayeur de parole et de description dans les techniques d'enquête utilisées au Cresson, a aussi changé. Considérée davantage comme un mode d'ancrage corporel aux terrains, c'est aux états de corps des chercheurs que nous avons prêté attention. Enfin, dans des contextes où la marche est pratiquée par les plus défavorisés, l'expérimenter avec nos collègues vénézuéliens et brésiliens a fait émerger, malgré notre position d'étranger, des problématiques sociopolitiques délicates à aborder. Si ces expériences corporelles partagées ont favorisé une connaissance réciproque, elles ont été aussi des embrayeurs de débat. A ce titre, la durée de la recherche (4 ans) et la répétition des séjours ont été importantes.

A Salvador, l'engagement dans le terrain a été contraint par l'ambiguïté de l'ambiance de Barra où à l'indolence du bord de mer se mêle la pesanteur d'une omniprésence policière et mafieuse, le risque latent d'agression, une violence interpersonnelle ténue. Le protocole d'enquête a toutefois articulé trois temps itératifs : faire corps, prendre corps, donner corpsaux ambiances urbaines (Thomas, 2010). "Faire corps » répond à une épistémologie de l'imprégnation. Entre 2011 et 2013, lors de séminaires ou d'ateliers pédagogiques, douze marches urbaines collectives d'une heure environ (de jour, de nuit, à différentes saisons)furent réalisées avec nos partenaires brésiliens ${ }^{4}$. Plusieurs groupes de 4 à 5 chercheurs se sont immergés dans les ambiances de Barra jusqu'à se laisser «embarquer» par elles et certaines des marchesont été réalisées en situation de handicap visuel, la privation de la vue aiguisant les autres sens.Cette immersion sensorielle procède d'une socialisation minimale au terrain : le corps du chercheur, «en prise » avec les stimulations sensorielles de l'environnement, constitue 
un instrument de captation de la vie sensible du quartier. «Prendre corps » répond à une épistémologie de l'incorporation. Son mouvement tient de la capacité du chercheur à être affecté par son terrain et à se laisser éprouver par son ambiance au point de réajuster ses gestes et perceptions. Dans un équilibre fragile entre acuité et lâcher-prise, cette porosité à l'environnement engage la description des régimes d'attention mais aussi celle des attitudes corporelles et des émotions. A ce titre, tout en constituant un moyen d'intégration des normes sociales régissant les coprésences (Duvoux, 2014), les émotions vécues au sein de notre groupe de marcheurs ont aussiopéré comme révélateurs de l'arrière-plan sociopolitique à l'œuvre dans le partage de l'espace.Enfin, «donner corps »répond à une épistémologie de la traduction. Il repose sur l'idée que rendre intelligible les dynamiques entre corps et ambiance nécessite des outils de description pluriels. Des temps de restitution ont ainsi été organisésà l'issue de chaque marche urbaine ${ }^{5}$, durant lesquels chaque groupe a rendu compte de ses impressions à l'aide de discours, croquis, mimesou performances dansées.

A Caracas, les marches ont consisté en des parcours récurrents effectués par les chercheurs (à 2, 3 ou 4) à travers la Redoma de Petare, sur plusieurs jours et en différents moments (en particulier aux heures de pointe), mais sur le seul été 2012et selon un protocole moins formalisé qu'au Brésil.Plus encore qu'à Salvador, l'expérience $\mathrm{du}$ terrain pose ici la question de l'enquête sous contraintes, du fait de la non connaissance préalable du lieu pour une partie de l'équipe, de notre mission limitée à une semaine et d'un contexte d'insécurité prégnant.Si Caracas s'avère l'une des villes les plus dangereuses au monde ${ }^{6}$, la réputation de la Redoma est aussi particulièrement négative, liée à une densité du lieu propice aux vols, au rôle de réseaux mafieux dans le contrôle du commerce informel et à la proximité des barrios d'où est principalement issue la violence meurtrière qui touche la ville. Notre appréhension du terrain a ainsi été largement filtrée par nos partenaires locaux (collègues, interlocuteurs municipaux) et leurs mises en garde quant au fait de nous rendre seuls sur place. La pression de l'insécurité nous a alors amenés à abandonner les observations initialement prévues en complément des parcours et à ne jamais nous arrêter lors de nos marches, afin d'éviter de nous surexposer aux vols ou aux agressions. Depar notre couleur de peau, notre tenue, et parce que la Redoma est surtout fréquentée par les classes populaires, il nous était impossible de nous «fondre dans le décor». De même, il nous a été très difficile de documenter le terrain par des enregistrements : impossible de faire des vidéos, matériel de prise de son nécessairement camouflé, photos prises à la sauvette. Les compte-rendu, immédiatement rédigés après chaque parcours, furent alors notre principal support d'analyse. Ils mettent l'accent sur la manière dont nous avons été affectés par le terrain, parlent de nos sentiments, humeurs, attitudes (individuelles et au sein du groupe en marche), de nos formes d'attention et d'engagement dans les situations.

\section{CE QUE L'ATTENTION AUX ETATS DE CORPS DU CHERCHEUR DEPLACE DANS L'ANALYSE}

Que peut-on alors apprendre de lieux aussi complexes en quelques semaines de terrain ? Quelle pertinence et quel sens accorder à des données basées sur le seul point de vue de chercheurs étrangers, dégagés des contraintes de la vie quotidienne ?Si regardé sous l'angle méthodologique de l'ethnographie, notre travail de terrain présente des limites, la réponse à ces questions tient pour nous dans l'attention particulière portée à l'expérience corporelle du chercheur et à son rôle dans le déplacement de l'analyse. D'emblée incluse dans le choix de protocoles de marche destinés à éprouver la ville à pied, cette attention 
a aussi été retravaillée in situ, par les conditions d'enquête. L'intérêt de l'enquête vénézuélienne tient ainsi au rapport d'étrangeté aux lieux des chercheurs français et à uneexpériencedu dépaysement ${ }^{7}$ qui, prédisposant à un déplacement par rapport à «ce qui va de soi », amène à rendre plus prégnants des phénomènes ou des perceptions routiniers pour des usagers familiers. Le bénéfice de l'enquête brésilienne tient davantage dans la tension entre familiarité, attachement aux lieux et fragilisation des enquêteurs. Tout en favorisant l'incorporation des rituels sociaux, la répétition des séjours a aussi diminué la vigilance des chercheurs qui n'ont pas su éviter l'agression, ont du gérer les émeutes urbaines d'avril 2014 et le coût émotionnel d'une assignation à résidence. Or, ces épisodes de trouble, de tension, d'épreuves, ont présenté pour chaque terrain un intérêt heuristique : celui de rendre intelligible des moments critiques dans l'ambiance du quartier, révélateurs de son arrière-plan sociopolitique.

Un autre point important concerne le recours à la notion d' «états de corps », développée en danse par Guisgand (2004) pour penser l'empathie motrice à l'œuvre entre danseurs et public au cours d'un spectacle. Ne procédant ni du geste ni de l'émotion, l'état de corps se décrit pour Guisgand comme un élan vital portés par les corps, une capacité à «tendre vers » rendant possible des manières d'éprouver et d'être ensemble. Si transposée à ce «moment partagé en commun» (Grout, 2012) qu'est l'espace public la notion offre matière à reconsidérer les dynamiques de coprésence à partir des états de corps des piétons, c'est plus précisément à partir des états des corps des chercheurs que nous avons tenté de montrer comment nos terrains, pour conflictuels qu'ils soient, peuvent aussi se décrire sous des formes sensibles partageables.Pour exemple, ceux-ci, soumis à des basculements dont la rapidité et l'intensité vont du déséquilibre à la tension, font passer d'un état de vigilance inquiète à un état de vigilance intense. Ces basculements, subtils à saisir, relèvent de ruptures, de passages graduels d'une ambiance à l'autre ou d'équilibre précaire de l'ambiance. A Barra, ilssont liés à l'intensification des processus de pacification ou à la mise en défaut de l'arrière-plan sécuritaire. C'est le déploiement inattendu de patrouilles militaires ou de policiers en civils arméset leur soudaine visibilité qui alerte. C'est l'arrivée massive de groupes de trafiquants ou d'habitants des rues qui met en tension, suscitant collectivement une anxiété, voire une peur intense.Pour autant, le collectif n'est pas mis à mal par ces états. La vigilance se partage. Elle rend palpable un mode de participation à l'espace de l'ordre de la dissension.A Caracas, l'état de vigilance est renforcé par notre difficulté à interpréter immédiatement certaines situations,à réagir rapidement et par notre manque de familiarité avec des codes là aussi marqués par l'arrière-plan insécuritaire. Il se révèle dans les moments de trouble où l'incertain est subitement perçu comme dangereux: lorsque le regard franc et direct d'un vendeur fait prendre conscience de sa propre vulnérabilité ou que la gêne éprouvée à la vue d'un jeune homme en train de se droguer suscite, après quelques pas hésitants, une posture faussement détendue. Au moment aussi de traverser une avenue où circulent dangereusement les véhicules, alors que dans une confusion sensorielle et motrice se mêlent la frayeur de frôler l'accident et l'angoisse d'avoir perdu de vue les collègues qui marchent en avant.

Autre exemple : l'état de saturation, lié à une surcharge sensorielle.A Barra, cet état est le fait d'un « remplissement » visuel : la prégnance des couleurs et des textures limite la perspective et gêne la discrimination à distance des scènes ordinaires. Les ardeurs du soleil accaparent, la chaleur brûlante freine la marche, la lumière éblouissante aveugle. L'odeur de la mer, de l'huile de palme frite, des camions poubelles attire, écœure, fait que l'on se « remplit les poumons » ou que l'on « traverse en apnée ». Ce sont aussi ces corps si proches de soi sur le trottoir qu'il faut ne pas toucher, suscitant parfois des 
sensations de malaise ; ces sifflements et sourires accrocheurs dont il faut se défaire tout en anticipant sa conduite ; cette extrême exposition de soi qu'il faut gérer. C'est cette acuité permanente qui accompagne une marche chaotique, faite de détours, d'accélérations, de piétinements, d'évitements répétés.Immergés dans un mouvement incessant de passants, commerçants et véhicules extrêmement bruyants, la Redoma offre le même sentiment étourdissant de pénétrer dans un monde «en soi ».Dans les deux cas, l'état de saturation tient à l'hyperesthésie des lieux : perception de l'intensité, de la profusion et du débordement y sont liés à la densité des flux, à la multitude des activités et micro-évènements qui se déroulent simultanément. Ce sont ainsi les bruits de freins, de klaxons, les rugissements des moteurs, les crissements de pneus ou les musique tonitruantesqui font sursauter, saturent jusqu'à empêcher toute concentration ou échange verbal. L'état de saturation expose donc le piéton à une surabondance sensible qui peut être de l'ordre de l'excès comme de la plénitude et n'empêche pas la coprésence.Defait, cet état questionne la qualité d'hospitalité du quartier (Joseph, 1997), sa capacité à accueillir dans un même espace-temps des corps en mouvement soumis à des pressions constantes et dont les objectifs, les rythmes, les «manières de marcher » diffèrent.

\section{DES ANALYSES QUI NE SONT PAS ACRITIQUES}

La compréhension par corps de ces situations de labilité ou de pacification nous engage sur la piste d'une critique sensible des urbanités contemporaines. Les analyses produites sur chaque terrain, en s'enracinant dans des émotions singulières mais partagées, mettent toutes à jour la pluralité des modes d'habitation sensibles du monde urbain. Plus qu'une vision déterministe et figée de l'urbanité, qui ferait valoir les effets néfastes de l'appropriation excessive ou de la pacification sur le déploiement de l'être-ensemble, l'analyse «par corps » en révèle la plasticité. Mobilisant l'idée d' « empathie motrice », la notion d'état des corps questionne en effet les modalités de constitution d'un commun en termes sensibles. En cela, elle renvoie indirectement à ce que Rancière, dans une tentative d'appréhension esthétique de la politique, nomme le "partage du sensible » (Rancière, 2000). La thèse est particulièrement intéressante puisqu'elle ne fait pas dépendre l'existence d'un commun et de formes de communauté d'une symbiose entre les éléments en présence. Elle montre davantage en quoi le conflit, le dissentiment, le retrait participent de l'instauration d'une communauté. De ce point de vue, questionner la manière dont se joue ce partage du sensibleengage une réflexion sur la façon dont certaines pratiques - artistiques chez Rancière, urbanistiques ici - reconfigurent l'exercice même de l'être-ensemble.

Dans cette perspective, nous avons reconduitsur nos deux terrains le protocole des marches collectives avec une vingtaine de personnes (universitaires, étudiants, architectes et acteurs municipaux). Certains professionnels présents, bien que travaillant sur la gestion des sites ou leur réaménagement, ne les fréquentaient que très rarement à pied, voire les évitaient.Prédisposant à une posture de disponibilité et d'empathie pour le lieu et entre les participants, marcher ensemble a constitué en soi la première forme de partage. La dimension corporelle a joué un rôle particulier, l'hyperesthésie ressentie s'offrant comme un préalable commun. Décentrant leur pratique quotidienne, cette expérience sensible a conduit la plupart des participants à décaler leurs représentations des terrains - jusque là stigmatisées à la Redoma de Petare sous l'expression du "chaos" ou à Porto da Barra sous une figure mémorielle immuable - en même temps que le partage d'autres perceptions a servi d'embrayeur de débat sur l'identité des lieux et sur les enjeux de leur transformation. 
${ }^{1}$ Thomas R. \& al. 2014, MUSE : Les énigmes sensibles des mobilités urbaines contemporaines. Agence Nationale de la Recherche, Programme «Espace et Territoire» édition 2010, Cresson.Un site web abondamment illustré donne à voir les matériaux d'enquête : http://www.anr-muse.fr.

${ }^{2}$ Descriptions issues d'observations menées entre septembre 2010 et juin 2013.

${ }^{3}$ Appliquées dès 2008 par l'Etat de Rio, ces politiques cherchaient à contrer le crime organisé dans les favelas.

${ }^{4}$ Laboratorio Urbano, Universidade Federal da Bahia

${ }^{5} \mathrm{http} / / / \mathrm{www}$. anr-muse.fr, rubrique Enquêter.

${ }^{6} \mathrm{http} / / /$ observatoriodeviolencia.org.ve/ws/informe-del-ovv-diciembre-2013-2/

${ }^{7}$ Thibaud J.P.2009, http://www.ambiances.net/seminars/lyon-grenoble-paris-2009-experience-dudepaysement.html

\section{BIBLIOGRAPHIE}

Duvoux N. 2014, « La peur de l’ethnographe » dans Genèses, n97 : 126-139.

Fiori S. \&Thomas R. 2014, «Être piéton à Salvador de Bahia et Caracas : une proximité fragilisée ? »dansGéoregards, $\mathrm{n}^{\circ} 6:$ : 83-96.

Grout C.2012,L'horizon du sujet. De l'expérience au partage de l'espace, Bruxelles, La Lettre Volée.

Guisguand P. 2004, « Pollock et les états de corps du peintre » dans DEMéter, Lille, Université de Lille 3, http://www.univ-lille3.fr/revues/demeter/corps/guisgand.pdf.

Joseph I.1997, «Prises, réserves, épreuves » dansCommunication, n65 : 131-142.

Olivier de Sardan J.P. 2000, «Le je méthodologique. Implication et explicitation dans l'enquête de terrain » dansRevue Française de Sociologie, ${ }^{\circ} 41$ : 417-445.

Rancière J.2000,Le partage du sensible. Paris, La Fabrique.

Thomas R. \& al. 2014, MUSE: Les énigmes sensibles des mobilités urbaines contemporaines, Agence Nationale de la Recherche, Programme «Espace et Territoire » édition 2010, Cresson.

Thomas R. \& al.2010,L'aseptisation des ambiances piétonnes au XXIe siècle, PIRVE CNRS MEEDDM, Cresson.

Rachel Thomas Chargée de Recherche CRESSON - UMR CNRS/MCC/ECN 1563 Ambiances, Architectures, Urbanités École Nationale Supérieure d'Architecture de Grenoble

Sandra Fiori Maître Assistante LAURE - UMR CNRS 5600 Environnement, Ville et Société École Nationale Supérieure d'Architecture de Lyon 